\title{
MicroRNA-18a inhibits cell growth and induces apoptosis in osteosarcoma by targeting MED27
}

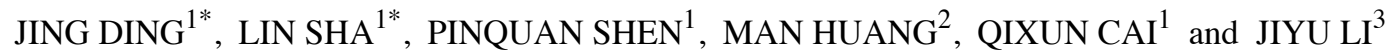 \\ ${ }^{1}$ Department of Pediatric Orthopaedics, Xinhua Hospital, School of Medicine, \\ Shanghai Jiao Tong University, Shanghai 200092; Departments of ${ }^{2}$ Good Clinical Practice, and ${ }^{3}$ General Surgery, \\ Shanghai Tenth People's Hospital of Tong Ji University, Shanghai 200072, P.R. China
}

Received December 6, 2017; Accepted April 4, 2018

DOI: $10.3892 /$ ijo. 2018.4374

\begin{abstract}
Osteosarcoma (OS) is a common malignant primary bone tumor and patients with OS are known to have a poor response to chemotherapy. MicroRNAs (miRNAs or miRs) are small non-coding RNA molecules (approximately 22 nucleotides in length) and they have recently become a topic for research as regards their role in cancer therapeutics. Previous studies have reported miR-18a expression in patients with OS is significantly decreased compared with that in normal adjacent tissue. miR-18a belongs to the miR-17-92 cluster encoded by the host gene MIRI7HG. However, the detailed role of miR-18a in OS remains to be determined. In this study, we demonstrated that miR-18a mimics inhibited MG63 and Saos-2 cell viability and migration. In addition, flow cytometry assay revealed that miR-18a induced OS cell apoptosis. Western blot analysis indicated that the expression levels of Bcl-2 and p-Akt were downregulated, while the levels of cleaved caspase- 3 and Bax proteins were upregulated by miR-18a. Moreover, we demonstrated that mediator complex subunit 27 (MED27) was the target of miR-18a through dual luciferase assay. Finally, data from in vivo experiments indicated that tumor growth in mice was significantly suppressed by miR-18a mimics, accompanied by a decrease in the percentage of Ki67-positive cells, and by the downregulation in MED27 and p-Akt protein expression levels. The findings
\end{abstract}

Correspondence to: Professor Qixun Cai, Department of Pediatric Orthopaedics, Xinhua Hospital, School of Medicine, Shanghai Jiao Tong University, 1665 Kongjiang Road, Shanghai 200092, P.R. China E-mail: caiqixun@xinhuamed.com.cn

Professor Jiyu Li, Department of General Surgery, Shanghai Tenth People's Hospital of Tong Ji University, 301 Yanchang Road, Shanghai 200072, P.R. China

E-mail: leejiyu@sina.com

${ }^{*}$ Contributed equally

Key words: microRNA-18a, osteosarcoma, cell apoptosis, phosphoprotein kinase B, mediator complex subunit 27 of the present study may aid in the clarification of the function of miR-18a, particularly as regards its role in the regulation of OS cell apoptosis, and indicate that MED27 may be a potential novel therapeutic target in the treatment of OS.

\section{Introduction}

Osteosarcoma (OS) is the most common malignant primary bone tumor, particularly in children and adolescents in both sexes (1). It has been reported that, from the epidemiological point of view, OS has a predilection for the metaphyseal portions of the long bone, as well as the distal femur and proximal tibia, which account for approximately $50 \%$ of all cases of OS (2). Furthermore, OS is a highly aggressive disease and the lungs are the primary target tissue for the metastasis of OS. Up to $25 \%$ of patients diagnosed with OS also suffer from lung metastases (3). OS mostly affects adolescents. In approximately three quarters of the cases, the age of patients with OS is between 15-25 years. The median age is 17 years, with a trend in the predominance of the male sex. There may be some correlation between OS and bone development, as during adolescence growth spurt, bone also grows rapidly (4). A relatively greater number of patients with OS have germline mutations in either the retinoblastoma or $p 53$ genes (5). This discovery indicates that these genes may be involved in the occurrence of the OS and provide us with some clues for future OS therapy. The clinical characteristics of OS include pain and swelling of the soft tissues, which are the most common symptoms of patients with OS (6). Currently, inpatient treatment for OS involves chemotherapy such as cisplatin, doxorubicin and methotrexate $(6,7)$. However, the 5 -year survival rate for patients treated with this chemotherapeutic regimen is only $70 \%$. In addition, $20 \%$ of patients with metastatic or recurrent disease exhibit chemoresistance, which limits the effectiveness of chemotherapy in malignant tumors, particularly OS (5-7). Thereby, it is urgent to investigate new pathways or to explore novel targets for the treatment of OS, particularly chemoresistant OS.

MicroRNAs (miRNAs or miRs) are single-stranded small RNAs which are approximately 22-24 nucleotides in length (8). They play an important role in the post-transcriptional regulation of gene expression. As miRNAs can bind to the complementary site of the 3'UTR sequence within the 
targeting mRNAs, they can mediate target RNA degradation or suppress translation (9-12). miR-18a is a member of the miR-17-92 family cluster, which includes 6 individual miRNAs: miR-17, miR-18a, miR-19a, miR-20a, miR-19b1 and miR-92a1, which are amplified in lymphomas and other cancer cells (13-15). This cluster and its paralogues play important roles in cancer development due to their ability to suppress the expression of a number of tumor-associated proteins, such as p53 and Akt (13-15), the overexpression of which promotes cell viability and reduces apoptosis by regulating cell cycle progression (16-19). The miR-17-92 cluster also plays an important role in normal growth and skeletal development. The deletion or duplication miR-17-92 or that of its paralogues can interrupt skeletal development, resulting in smaller embryos and post-natal death due to ventricular septal defects (20).

Recently, much attention has been paid to miRNAs, such as the miR-17 family, which has been reported to regulate tumor growth in various types of cancer. miR-18a has been reported to function as an onco-miRNA, promoting cell viability and facilitating tumor progression $(21,22)$. However, it has also been demonstrated that the abnormally high expression of miR-18a in gastric cancer tissues inhibits the expression of interferon regulatory factor 2 to modulate tumor protein p53 (TP53) expression (16). miR-18a promotes the viability of esophageal squamous cell carcinoma cells by increasing cyclin D1 expression (22). miR-18a expression has also been shown to be elevated in prostate cancer and to promote tumorigenesis through the suppression of serine/threonine-protein kinase 4 (STK4) in vitro and in vivo (23). In addition, miR-18a has been shown to be upregulated in glioblastoma tissues, enhancing cell viability and facilitating the exit of cells from cell cycle arrest (24).

Recently, it has been reported that miR-1, miR-9, miR-18a, miR-18b, miR-126, miR-133b, miR-144, miR-195 and miR-451 expression levels are consistently decreased in both cell lines and clinical samples of OS compared with normal bone tissues (25-27). According the study by Namløs et al in 2012, miR-18a expression in patients with OS was significantly decreased compared with that in normal adjacent tissue (25). However, the exact role of miR-18a in the development of OS remains to be determined. Therefore, in this study, we aimed to investigate the role of miR-18a in OS. We found miR-18a induced OS cell (MG63 and Saos-2 cells) apoptosis and suppressed cell migration and invasion. The results form our in vivo experiments using mice also demonstrated that miR-18a significantly inhibited tumor growth. Immunohistochemistry also confirmed that Ki67 and p-AKT expression was increased by miR-18a. Furthermore, mediator complex subunit 27 (MED27) was identified as a direct target of miR-18a with bioinformatics tools and validated in both OS cell lines.

\section{Materials and methods}

Cell culture. The human osteosarcoma cell lines, MG63 and Saos-2, were purchased from the Stem Cell Bank, Chinese Academy of Sciences (Shanghai, China). The MG63 cells were propagated in Dulbecco's modified Eagle's medium (DMEM; Thermo Fisher Scientific, Waltham, MA, USA) and the Saos-2 cells were cultured in RPMI-1640 medium (Sigma,
St. Louis, MO, USA). Both culture media were supplemented with $10 \%$ fetal bovine serum (Thermo Fisher Scientific) and gentamicin (40 $\mu \mathrm{g} / \mathrm{ml}$, Sigma). All the cells were cultured at $37^{\circ} \mathrm{C}$ with $5 \% \mathrm{CO}_{2}$ in a humidified cell culture incubator (Sanyo, Tokyo, Japan).

Transient cell transfection. A total of $3 \times 10^{5}$ MG63 cells and $3 \times 10^{5}$ Saos-2 cells were plated in 6-well plates and transfected with $100 \mathrm{nM}$ miR-18a mimics, $100 \mathrm{nM}$ miR-18a inhibitor or the scramble control, which were synthesized by Shanghai GenePharma Co. (Shanghai, China), using Lipofectamine ${ }^{\circledR} 2000$ reagent (Invitrogen/Thermo Fisher Scientific) according to the manufacturer's instructions. At $48 \mathrm{~h}$ following transfection, cell viability, and protein and mRNA expression levels were analyzed in each group of cells.

Cell viability assay. The Cell Counting kit-8 (\#C0038, Beyotime, Shanghai, China) was used to determine cell viability. According to the instructions of the manufacturer, the procedure was as follows: The MG63 or Saos-2 cells at the logarithmic growth phase were plated in a 96-well plate at a density of 3,000 cells/well. The cells were then transfected with $100 \mathrm{nM}$ miR-18a mimics, miR-18a inhibitor or the control and cultured for a $72 \mathrm{~h}$. At indicated time-points, $20 \mu \mathrm{l}$ cell counting kit solution was added to each wall, and the cells were further incubated for $0.5 \mathrm{~h}$ in a cell incubator. Moreover, the cells were transfected with $10,30,50,100$ or $150 \mathrm{nM}$ miR-18a mimics, the inhibitor or the control (mock) for $72 \mathrm{~h}$. The number of living cells was measured using a microplate reader (\#168-1130, Bio-Rad Laboratories, Hercules, CA, USA), at a $450 \mathrm{~nm}$ wavelength. The inhibitory rate $=1-$ the OD value of the miR-18a mimic transfected/OD value of the control group. The half maximal inhibitory concentration $\left(\mathrm{IC}_{50}\right)$ was then calculated.

Wound healing assay. The MG63 or Saos-2 cells were plated into 6-well plates ( $2 \times 10^{5}$ cells/well) and transfected with $30 \mathrm{nM}$ miR-18a mimics, miR-18a inhibitor or the control (mock). When the cells grew to $100 \%$ confluence, a vertical wound was made down through the cell monolayer using 1,000 $\mu \mathrm{l}$ pipette tip to press firmly and swiftly against the top of the tissue culture plate. The media and cell debris were carefully aspirated prior to further culture. The wound was captured under a microscope (Leica Microsystems, Inc., Buffalo Grove, IL, USA) at each indicated time-point. The snapshot image was used to analyze the distance of one side of the wound to the other side using a scale bar.

Transwell cell invasion assay. A total of $100 \mu 1$ of the MG63 or Saos- 2 cells $\left(1 \times 10^{6}\right.$ cells $\left./ \mathrm{ml}\right)$ transfected with $30 \mathrm{nM}$ miR-18a, miR-18a inhibitor or the control (mock) were plated on the top of the membrane in a Transwell insert into a 24-well plate. When the cells had settled down, $600 \mu 130 \%$ fetal bovine serum was added to the bottom of the lower chamber in a 24-well plate. The cells that did not migrate from the top of the upper membrane and remaining culturing media were removed carefully using cotton swabs, while the migrated cells into the bottom of the lower chamber in the well were fixed and stained with crystal violet (\#R40052, Thermo Fisher Scientific) at room temperature for $48 \mathrm{~h}$. The cells in different 
fields of view were counted under a microscope (\#CKX41, Olympus America, Inc., Center Valley, MA, USA) and using the average sum of cells to analyze the invasion rate.

Flow cytometric analysis of cell apoptosis. Cell apoptosis was measured using Annexin V-FITC and propidium iodide (PI) (Thermo Fisher Scientific) according to the manufacturer's instructions. Briefly, $1 \times 10^{6}$ MIA PaCa-2 cells were plated into 100-mm dishes (Corning, New York, NY, USA) and then transfected with miR-128 mimics or the mock control $(100 \mathrm{nM})$. After $72 \mathrm{~h}$, the cells were lysis, and stained with Annexin-FITC and PI prior to being subjected to flow cytometry (\#660344 flow cytometer, BD Biosciences, Franklin Lakes, NJ, USA). This assay can sort and identify the early apoptotic (Annexin $\mathrm{V}-\mathrm{FITC}^{+} / \mathrm{PI}^{-}$) cells, the primary necrotic (Annexin V-FITC $/ \mathrm{PI}^{+}$) cells, and late apoptotic (Annexin V-FITC ${ }^{+} / \mathrm{PI}^{+}$) cells. For each group, the samples were examined in triplicate.

Predicted target analysis of miR-18a. miRecords (http:// c1.accurascience.com/miRecords/) is an online database which can be used to predict the binding site of miRNA to its targets. It integrates the results of several online miRNA target prediction tools, including DIANAmicroT, miRanda, PicTar and TargetScan (28). This online software was used to predict the potential targets of miR-18a.

3'UTR-luciferase reporter gene assay. All vectors were purchased from Genewiz (Beijing, China), which carried MED27 containing the predicted miR-18a binding sites with the wild-type or mutant 3'UTR. The MG63 or Saos-2 cells were plated into a 24-well plate and transfected with MED27 vector or mutant vector using Lipofectamine ${ }^{\circledR} 2000$ transfection reagent (Thermo Fisher Scientific), according to the manufacturer's instructions. After $4 \mathrm{~h}, 100 \mathrm{nM}$ of miR-18a mimics or the control (mock) were transfected into the cells, respectively. After a further $48 \mathrm{~h}$ of culture, the cells were lysed and the luciferase activities were analyzed by a dual luciferase assay kit (Promega, Madison, WI, USA).

Reverse transcription-quantitative PCR (RT-qPCR). Total RNA was isolated from the MG63 or Saos-2 cells using the PicoPure $^{\mathrm{TM}}$ RNA Isolation kit (Arcturus, Sunnyvale, CA, USA) according to the manufacturer's instructions. Subsequently, $2 \mu \mathrm{g}$ of RNA were utilized for cDNA synthesis using SuperScript III RNase H Reverse Transcriptase (Thermo Fisher Scientific). Following the reverse transcription reactions, the miR-18a expression level was detected by microRNA assay (Thermo Fisher Scientific). The primer for GAPDH was as follows: forward, 5'-AATGCATCCTGCACCACCAA' and reverse, 5'-GTAGCCATATTCATTGTCATA'. The qPCR procedure was performed as follows: for the holding stage: Step 1, heating from $25^{\circ} \mathrm{C}$ to $95^{\circ} \mathrm{C}$ at the rate of $1.6^{\circ} \mathrm{C} / \mathrm{sec}$ and holding for $2 \mathrm{~min}$ at $50^{\circ} \mathrm{C}$; step 2 , heating from $50^{\circ} \mathrm{C}$ to $95^{\circ} \mathrm{C}$ at the rate of $1.6^{\circ} \mathrm{C} / \mathrm{sec}$ and then holding for $10 \mathrm{~min}$ at $95^{\circ} \mathrm{C}$. For the PCR stage: Step 1, initial denaturation at $95^{\circ} \mathrm{C}$ for $15 \mathrm{sec}$; step 2, annealing extension at $60^{\circ} \mathrm{C}$ for $1 \mathrm{~min}$. The temperature was cooled down from $95^{\circ} \mathrm{C}$ to $60^{\circ} \mathrm{C}$ at the rate of $1.6^{\circ} \mathrm{C} / \mathrm{sec}$ and the denaturation and extension were then repeated for 40 cycles. Ct was measured at the PCR stage with the by ViiTM 7 system (\#4458571, Bio-Rad Laboratories) Based on the real-time PCR results of Ct number, the expression of mRNA levels was calculated using the $2^{-\Delta \Delta C a}$ method (29) and normalized to the internal reference control, GAPDH.

Western blot analysis. The MG63 or Saos-2 cells were collected at $72 \mathrm{~h}$ following transfection with miR-18a mimics or the mock control. Tumor tissues were collected at the end of the experiment (5 weeks) and then total proteins were extracted by using $1 \mathrm{X}$ loading buffer. The protein concentration was quantified using the BCA Protein Assay kit (Thermo Fisher Scientific). A total of $30 \mu \mathrm{g}$ of protein were loaded per lane per group, and electrophoresis was performed on a 10\% Tris-SDS gel. Following electrophoresis, the gel was blotted onto polyvinylidene fluoride membranes (Thermo Fisher Scientific). For the following locking and antibody incubation, the iBind kit was used according the manufacturer's instructions. The primary antibodies used were as follows: MED27 (1:500; \#SAB1411657; Sigma), Bax (1:500; \#5023), Bcl-2 (1:500; \#2872), p-Akt (1:500; \#4060), Akt (1:500; \#4691), matrix metalloproteinase (MMP)2 (1:500; \#40994) and MMP9 (1:500; \#13667) (all from Cell Signaling Technology, Danvers, MA, USA). Cleaved caspase-3 (1:1,000; \#sc-98785) and GAPDH (1:1,500; \#sc-66163) were purchased from Santa Cruz Biotechnology, Inc., (Santa Cruz, CA, USA). The secondary antibody (horseradish peroxidase conjugated anti-rabbit) was provided from Cell Signaling Technology, Inc. (\#7074, 1:3,000). The signal was detected with super sensitive regent (Thermo Fisher Scientific), and the specific proteins were detected with the ChemDoc ${ }^{\mathrm{TM}}$ imaging system (Bio-Rad Laboratories). Quantification of the protein data was carried out using the density in the blots with Image Lab software version 4.0 (Bio-Rad Laboratories).

In vivo mouse tumor xenograft model. A total of 30 (5-6 weeks old, weighing 18-21 g) female BALB/c nude mice were purchased from Vital River Laboratories (Beijing, China) and housed in an SPF environment with a 12-h/12-h light/dark cycle at the Animal Center in Xinhua Hospital. These mice were supplied with free water and food and the temperature was maintained at $22 \pm 2^{\circ} \mathrm{C}$ with $40-70 \%$ relative humidity. Some of the MG63 cells were transfected with the lentivirus with the miR-18a sequence, while the other cells were only transfected with the lentivirus with mock sequence (Shanghai GenePharma Co.). The mice were subcutaneously implanted with these two modified MG63 cells and the tumor size and mouse body weights were monitored once a week for successive 5 weeks. The mice were allowed free access to food and water. The MG63 osteosarcoma cells transfected with the miR-18a mimics or the mock control lentivirus ( $5 \times 10^{7}$ in $100 \mu \mathrm{l}$ in PBS) were subcutaneously injected into the left flank of each mouse. It should be noted that a total of 30 mice were subcutaneously implanted with these the modified MG63 cells. However as the tumor formatting rate is approximately $70 \%$, we selected 20 mice with good tumor formatting for the experiment. The other 10 mice with no or very small tumors were not used. Thus, in the experiments, there were 10 mice per group. The tumor volumes (TV) were calculated using calipers once per week, which were calculated as follows: $\mathrm{TV}=\left(\right.$ width $^{2} \mathrm{x}$ length $) / 2$. 

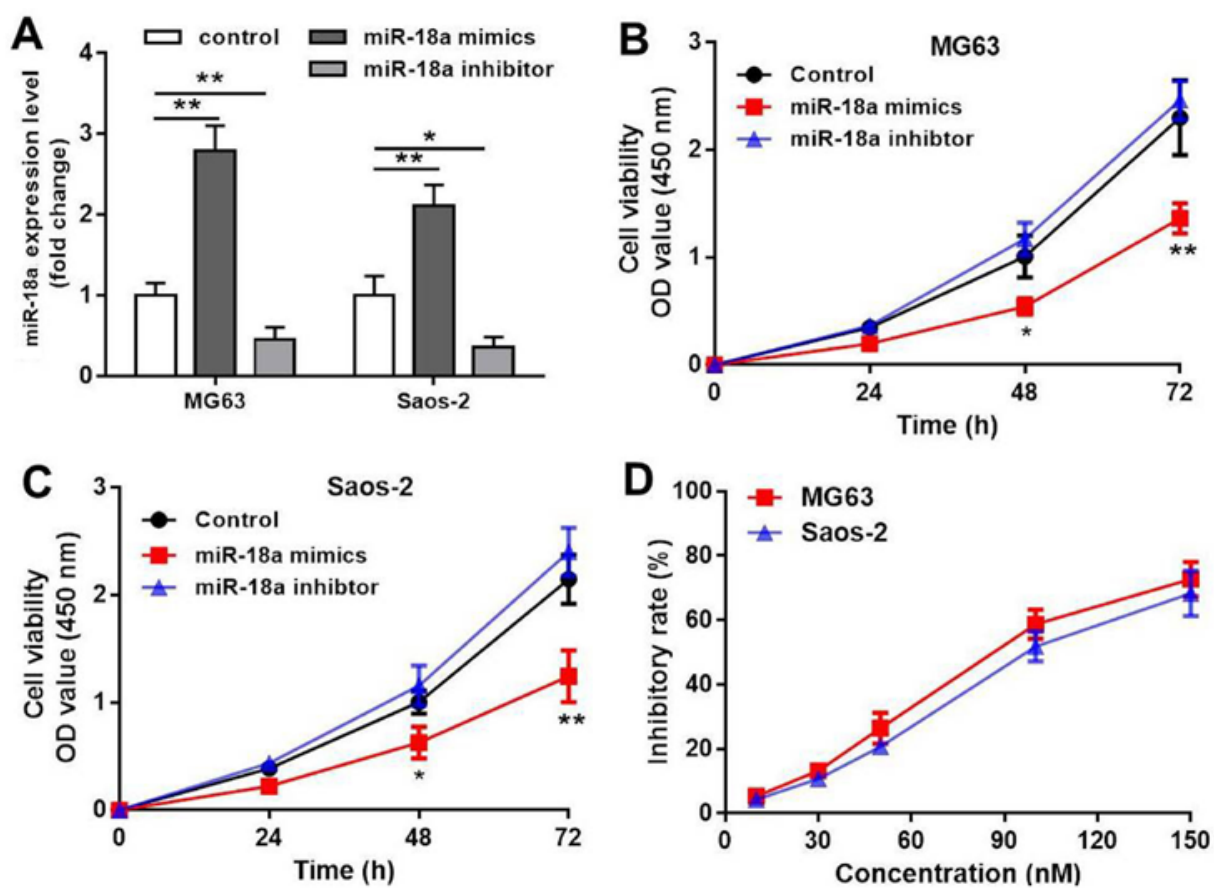

Figure 1. miR-18a inhibits the viability of both the MG63 and Saos-2 osteosarcoma cell lines. (A) Total RNA was extracted from the MG63 and Saos-2 cells with or without miR-18a mimics treatment. The levels of miR-18a were analyzed by RT-qPCR. For all the experiments, the miR-18a mRNA expression levels were normalized to the internal gene, GAPDH. Each primer was in run triplicate $\left({ }^{*} \mathrm{P}<0.05,{ }^{* *} \mathrm{P}<0.01\right)$. (B and C) Transfection with $100 \mathrm{nM}$ miR-18a mimics exerted antitumor effects on the MG63 and Saos-2 cells by inhibiting their viability in a time-dependent manner $\left({ }^{*} \mathrm{P}<0.05,{ }^{* *} \mathrm{P}<0.01\right)$. (D) The cells were transfected with 10,30,50, 100 or $150 \mathrm{nM}$ miR-18a mimics for $72 \mathrm{~h}$.

Both tumor width and length are presented in $\mathrm{mm}$. At the end of the experiment, the mice were sacrificed. The tumors were then excised from the mice and weighed. A portion of tumors was fixed into 10\% PFA as soon as possible for further use. Another portion of tumors was lysed for western blot analysis. The maximum diameter of a single tumor was $15.12 \mathrm{~mm}$ and the maximum tumor volume was $727.54 \mathrm{~mm}^{3}$ in our study. No mouse developed multiple tumors. All the above-mentioned procedures using these nude mice were approved by the Xin Hua Hospital Animal Experimental Ethics Committee (Approval no. 201703678).

Immunohistochemistry (IHC) assay. Ki67 as a proliferation protein was used to detect the cell proliferation in the tumor tissues isolated from the mice. All tissues were fixed with formalin and paraffin-embedded in advance means immediately when the tissue samples were isolated from the mouse bodies. They were then stored for use at $-80^{\circ} \mathrm{C}$ or deparaffinized, rehydrated and then immersed in a target retrieval solution ( $\mathrm{pH}$ 6.0), and boiled at medium baking temperature 3 times for $10 \mathrm{~min}$ once in a microwave. They were then incubated with $3 \%$ BSA for $1 \mathrm{~h}$ and then incubated with primary antibody against Ki67 (1:500; \#701198, Thermo Fisher Scientific) for $1 \mathrm{~h}$. After washing with PBS 3 times, the samples were then incubated with biotinylated secondary antibody (1:8,000; \#65-6140, Thermo Fisher Scientific) followed by the addition of horseradish peroxidase-conjugated streptavidin (\#N100, Thermo Fisher Scientific). For TUNEL assay, the procedure was carried out according to the instructions of the manufacturer (Roche Diagnostics, Indianapolis, IN, USA). The samples were counterstained with hematoxylin and the target-positive cells were counted in 3-4 different fields and photographed using the EVOS ${ }^{\mathrm{TM}}$ FL Auto Imaging System (Thermo Fisher Scientific).

Statistical analysis. Statistical analyses between 2 groups and multiple groups were carried out using a two-tailed Student's t-test and one-way ANOVA followed by Dunnett's test, respectively (GraphPad Prism7, La Jolla, CA, USA). In all the assays, a P-value $<0.01$ was considered to indicate a statistically significant difference. All experiments were performed in triplicate, except for the tissue samples from the tumor xenograft model for IHC and all values are presented as the means \pm SD .

\section{Results}

miR-18a inhibits MG63 and Saos-2 cell viability. Firstly, we transfected the MG63 and Saos-2 cells with miR-18a mimics or miR-18a inhibitor and confirmed that the miR-18a levels were significantly upregulated or downregulated, respectively $(\mathrm{P}<0.01$, Fig. 1A). Subsequently, the effects of miR-18a on OS cell viability were measured by CCK8 assay. The results revealed that transfection with $100 \mathrm{nM}$ miR-18a mimic inhibited MG63 and Saos-2 cell growth in a time-dependent manner $(\mathrm{P}<0.01$, Fig. $1 \mathrm{~B}$ and $\mathrm{C})$. In addition, the $\mathrm{IC}_{50}$ values of the miR-18a-transfected MG63 and Saos-2 cells were 88.6 and $95.8 \mathrm{nM}$, respectively (Fig. 1D), which indicated that $100 \mathrm{nM}$ of miR-18a appeared to be cytotoxic.

miR-18a mimics inhibits OS cell migration and invasion, and induces cellular apoptosis. Wound healing assay was used to examine the effects of miR-18a on OS cell migration. As 

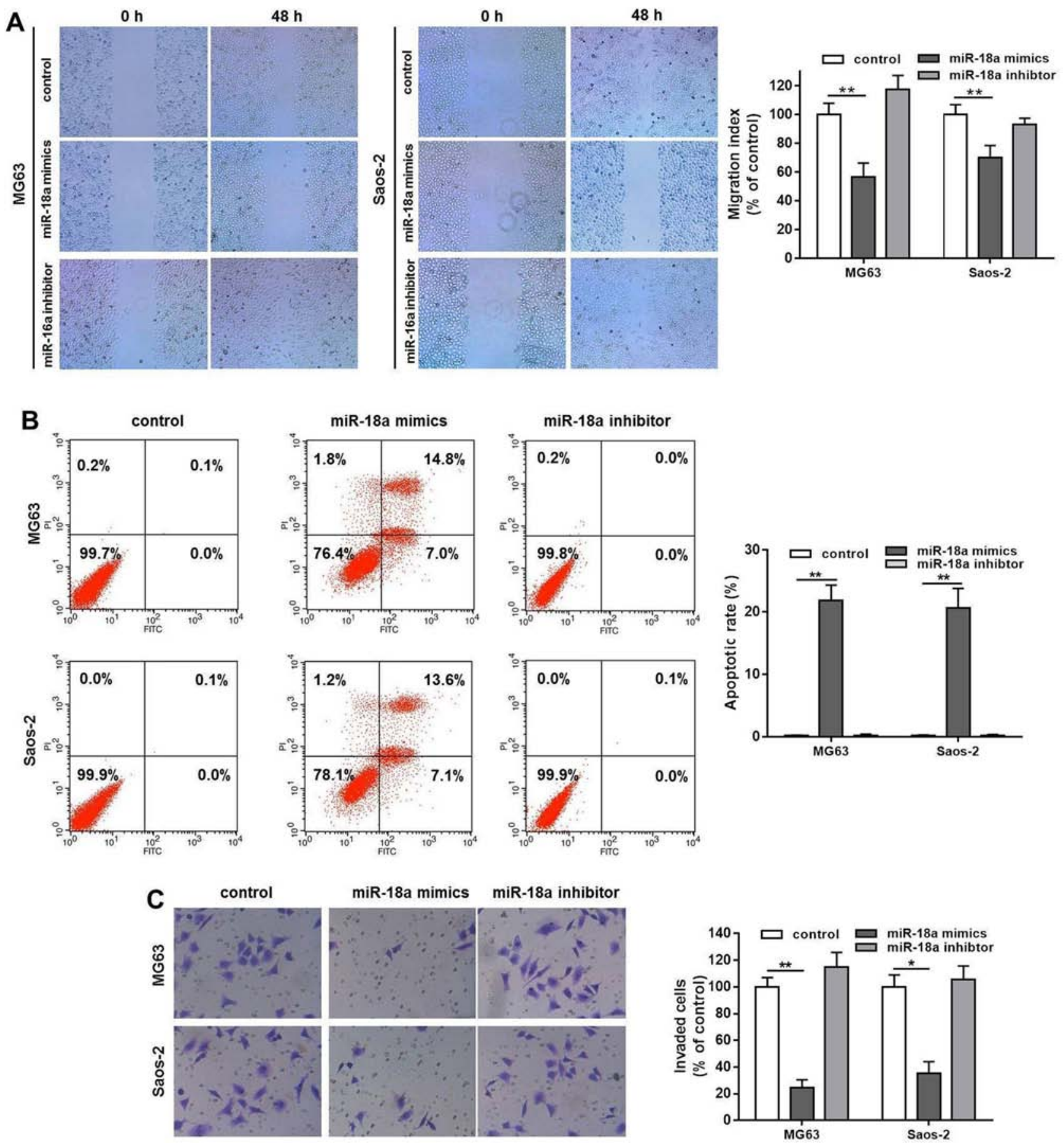

Figure 2. miR-18a inhibits cell migration and invasion, and induces the apoptosis of MG63 and Saos-2 osteosarcoma cells. (A) miR-18a inhibited MG63 and Saos-2 cell migration (x20 magnification) at $48 \mathrm{~h}$. Bar charts show the results of the quantification of the migration percentage compared with the control. (B) miR-18a induced MG63 and Saos-2 cell apoptosis. PI and Annexin V-FITC/PI double staining of the apoptotic cells. The quantification of the early and late apoptotic cells was calculated. (C) miR-18a inhibited MG63 and Saos-2 cell invasion (x20 magnification). Bar charts show the results of the quantification of the invasion percentage compared with the control $\left({ }^{*} \mathrm{P}<0.05,{ }^{* *} \mathrm{P}<0.01\right)$.

shown in Fig. 2A, transfection with miR-18a mimics markedly inhibited MG63 cell migration and invasion, whereas transfection with miR-18a inhibitor had no effect and the inhibitory effect of miR-18a mimcs on Saos- 2 cell migration was relatively weak, but compared with the control there was still a difference. This might due to the rapid proliferative ability of the Saos- 2 cells. In addition, the results of flow cytometry assay indicated that transfection with miR-18a mimics increased Annexin V-FITC/PI double staining positive rate in both the MG63 and Saos-2 cells (Fig. 2B), which indicated that miR-18a significantly induced MG63 and Saos-2 cell apoptosis compared with the control (Fig. 2B). Similar to the results of wound healing assay, the results of Transwell assay demonstrated that transfection with miR-18a mimics blocked the invasive ability of the MG63 and Saos-2 cells (Fig. 2C). The invasion rate in the control group was considered as $100 \%$, while this rate was decreased to only 20 or $30 \%$ following transfection of the cells with miR-18a mimics (Fig. 2C). 
A

\begin{tabular}{|c|c|c|c|c|c|c|c|}
\hline MicroRNA & Gene & Ref seq ID & Seed Length & Start & Sequence & End & Region \\
\hline hsa-miR-18a & MED27 & NM_004269 & 7 & 999 & AAGGUGC & 993 & 3 UTR \\
\hline
\end{tabular}
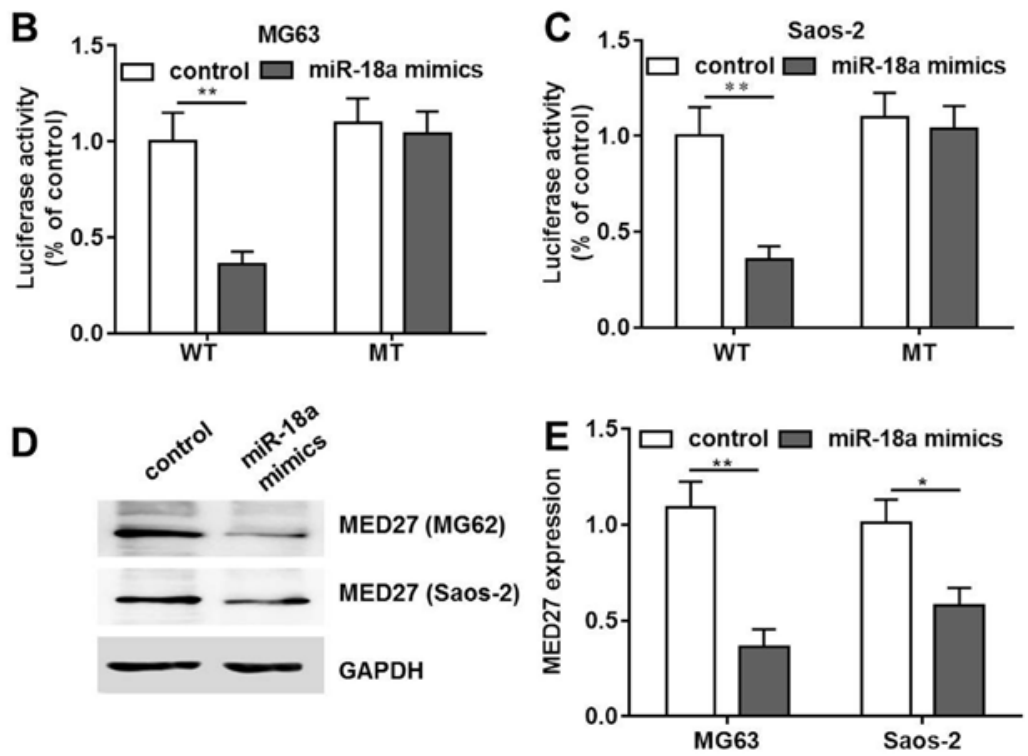

Figure 3. MED27 is a direct target of miR-18a. (A) Gene structure of MED27 showing the predicted target site of miR-18a in its 3'UTR with the sequence of AAGGUGC. (B and C) MG63 and Saos-2 cells were transfected with the reporter gene containing wild-type or mutant MED27 sequence. In both the MG63 and Saos-2 cells, miR-18a only significantly blocked the luciferase activity of the cells, which were transfected with wild-type MED27 (** $<0.01$ compared with the control group). (D) miR-18a downregulated MDE27 protein expression in both the MG63 and Saos-2 cells. (E) Quantification of MED27 protein expression $\left({ }^{*} \mathrm{P}<0.05,{ }^{* *} \mathrm{P}<0.01\right.$ compared with the control group).

miR-18a mimics induce OS cell apoptosis by targeting MED27. Bioinformatics tools were used in order to investigate the antitumor mechanisms of miR-18a in the MG63 and Saos-2 cells. The use of the online bioinformatics tools, TargetScan (http://www.targetscan.org/vert_61/) and miRanda (http:// www.microrna.org/microrna/) predicted MED27 as a target of miR-18a with experiments were carried out to confirm whether MED27 was a direct target of miR-18a (data not shown). Two plasmids was constructed as follows: One was wild-type MED27 and the other was the mutant with the MED27-3'UTR mutation. Both plasmids were fused with a luciferase reporter gene (Fig. 3A). The results of luciferase assay using the OS cells (MG63 and Saos-2) indicated that transfection with miR-18a mimics significantly inhibited the luciferase activity of the wild-type construct. However, no difference was observed between the control and the miR-18a-trasnfected group with the MED27 mutant. Moreover, the results of both cell lines revealed comparable activity (Fig. 3B and C). The results of western blot analyses also confirmed that transfection with miR-18a mimics downregulated MED27 protein expression in both the MG63 and Saos-2 cells (Fig. 3D and E). These findings indicated that MED27 was the direct target of miR-18a in the OS cells.

miR-18a mimics induce OS cell apoptosis via the caspase-3 dependent pathway. In order to further investigate the antitumor mechanisms of miR-18a, we examined the expression of several proteins, including Bax, Bcl-2, p-Akt and cleaved caspse- 3 in the MG63 and Saos- 2 cells. The results indicated that transfection with miR-18a mimics downregulated Bcl-2 and p-Akt expression (Fig. 4A-C), which are the negative regulators of cellular apoptosis. We also found that transfection with miR-18a upregulated Bax and cleaved caspse-3 expression, which are the promoters of cellular apoptosis (Fig. 4A, D and E). In addition, transfection with miR-18a mimics inhibited OS cell migration and invasion via the downregulation of MMP2 and MMP9 (Fig. 4A, F and G)

miR-18a mimics inhibit tumor growth in a MG63 tumor xenograft model. In order to determine whether miR-18a inhibits OS tumor growth activity in vivo, lentivirus-infected MG63 cells were established and injected into nude mice. From the results, it was found that the tumors derived from the cells transfected with the miR-18a mimics grew at a significantly slower rate compared with the lentivirus control cells over a period of 4 weeks. The tumor size in the miR-18a lentivirus group ranged from 280 to $320 \mathrm{~mm}^{3}$ compared with that in the control group, which ranged from 500 to $700 \mathrm{~mm}^{3}$ over a period of 5 weeks (Fig. 5A). At the end of the experiment, the tumor weight in the lentivirus miR-18a group was only $0.7 \mathrm{~g}$ compared with that in the control group which was approximately $1.6 \mathrm{~g}$ (Fig. 5B and C). Moreover, no obvious changes in body weight were observed in the 2 groups (Fig. 5D).

Effects of miR-18a mimics on Ki67, TUNEL and MED27/p-Akt expression levels in tumor xenografts. To further validate the antitumor activity of miR-18a on OS tumor xenogafts, we examined the Ki67 and TUNEL positive cell rate by IHC. The results revealed that the number of Ki67-postive cells decreased by $20 \%$ and that of TUNEL-positive cells increased by approximately $20 \%$ in the lentivirus miR-18a group compared with the lentivirus control (Fig. 6A-D). We also confirmed that miR-18a significantly inhibited MED27 
A

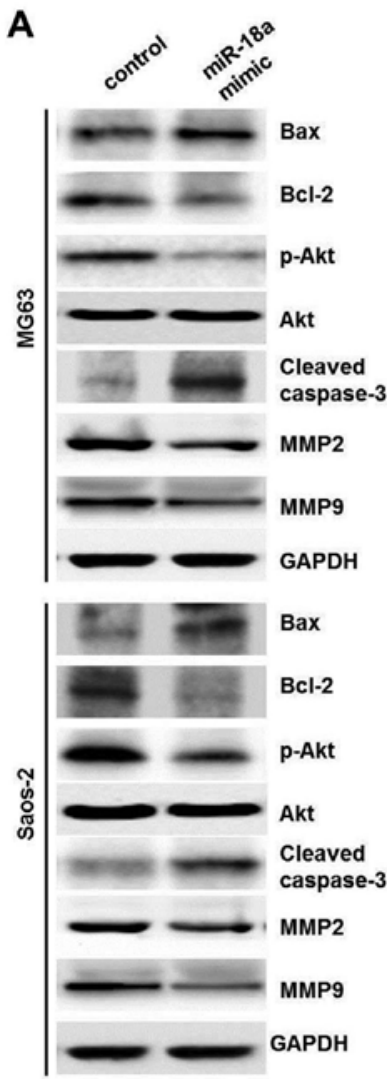

B
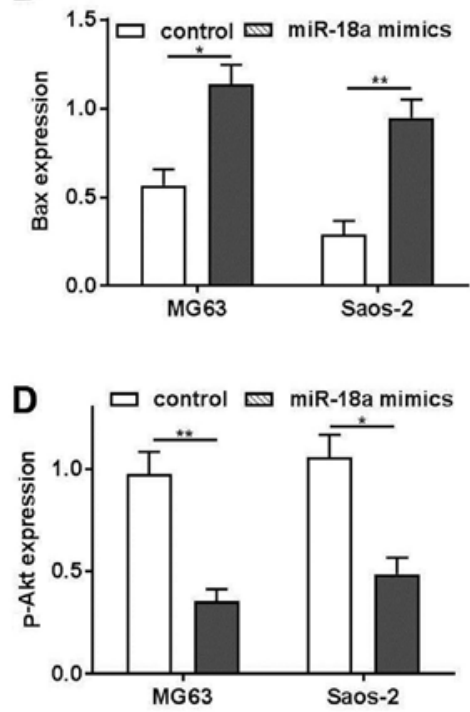

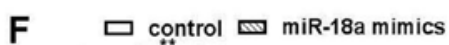

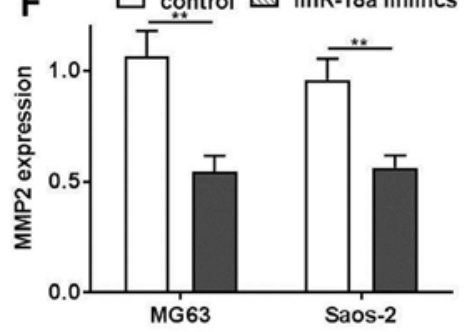

C
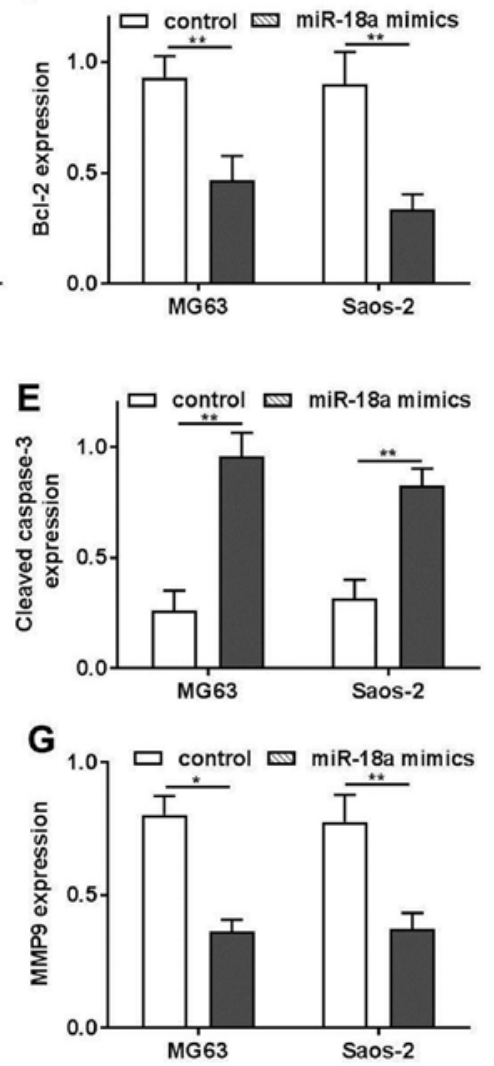

Figure 4. miR-18a induces apoptosis by enhancing Bax and cleaved caspase-3 protein expression, and decreasing Bcl-2 and p-Akt protein expression in both the MG63 and Saos-2 cells. (A) The expression levels of Bax, Bcl-2, p-Akt and cleaved caspase-3 in the MG63 and Saos-2 cells were detected by western blot analysis. (B-G) Quantification of the proteins levels of Bax, Bcl-2, p-Akt, cleaved caspase-3, MMP2 and MMP9 in both the MG63 and Saos-2 cells (*P<0.05, $\left.{ }^{* *} \mathrm{P}<0.01\right)$.

and p-Akt expression in vivo, which was consistent with the findings in vitro (Fig. 6E-G).

\section{Discussion}

Cancer is not only one of the main causes of death worldwide, but is also one of the most rapidly growing causes of death $(2,12)$. OS mainly affects adolescents and $70 \%$ of patients with OS succumb to the disease due to OS metastasis and chemoresistance $(6,7)$. Thus, it is mandatory to develop novel targets or markers for the efficient treatment of OS. Over the past decades, miRNAs have been identified by researchers as tumor markers; however, the journey from bench to bedside is still a long one, and it may take time for miRNAs to be used as targets in clinical practice (28-30). miRNAs are a group of small non-coding RNAs (ncRNAs) that post-transcriptionally regulate gene expression by targeting the 3'UTR of their corresponding mRNAs. Dysregulated miRNAs have been regarded as a novel type of 'onco-miRNAs' or 'tumor suppressors', which may play essential roles in cancer initiation, progression and metastasis (12,31-33).

miR-18a belongs to a large miRNA cluster known as the miR-17-92 cluster, which encodes a total of 5 miRNAs, including miR-17, miR-19a, miR-20a, miR-19b and miR-92a. A number of studies have focused on the regulation of miR-17-92 in multiple types of tumors from lymphomas to solid tumors (20-24,32). Moreover, it has been reported that the miR-17-92 gene cluster transcript can be activated by the c-myc, N-myc and E2F families $(34,35)$. Of noted, the expression levels of each miRNA in the cluster are not exactly parallel with each other, suggesting that the processing or stability of the miRNAs is differentially regulated (33). It has been previously reported that miR-17-92 plays an essential role in the progression and development of breast cancer and that the overexpression of miR-17 promotes human breast cancer cell migration and invasion through the downregulation of HMG-box transcription factor 1 (HBP1), which is the regulator of miR-17 stability $(36,37)$. Furthermore, it has been demonstrated that miR-18a reduces DNA damage repair signaling and increases cellular radiosensitivity (37).

In the present study, we demonstrated that miR-18a inhibited the viability, migration, and invasion, and induced the apoptosis of the MG63 and Saos-2 cells. In vivo experiments also confirmed that miR-18a suppressed tumor growth and that this was accompanied by a decrease in the Ki67-positive cell rate, a decrease in Bcl-2 and p-Akt expression, and by the upregulation of Bax. The above-mentioned findings support the notion that miR-18a functions as a tumor suppressor. In addition, the results of bioinformatics analysis and luciferase assay validated MED27 as the direct target of miR-18a.

Given the demonstration of the mutations or overexpression of some MED proteins in various human cancers (38-40), MED proteins have been recognized to play an increasingly essential role in tumorigenesis and development. Although 

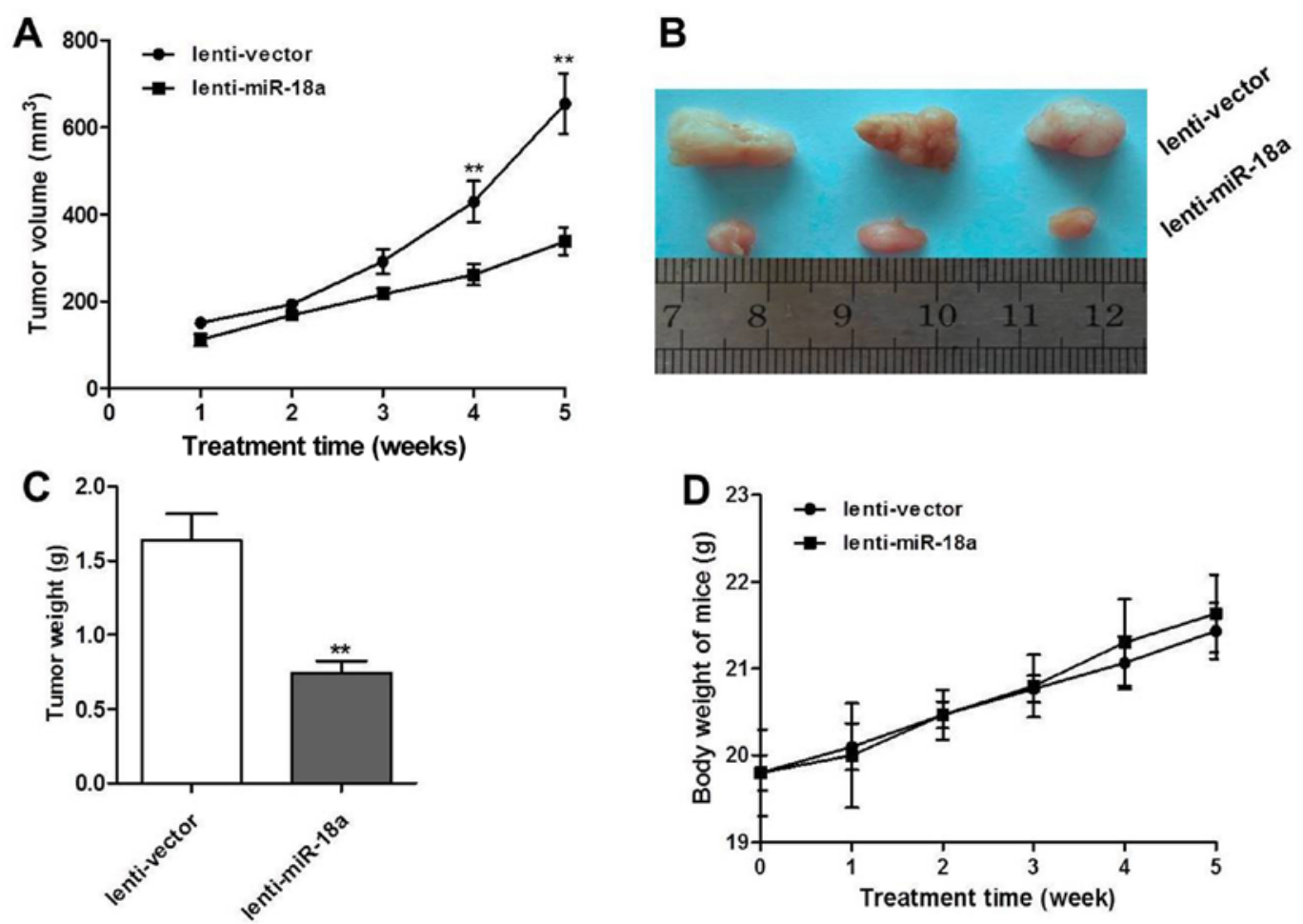

Figure 5. miR-18a inhibits tumor growth in a MG63 tumor xenograft model. (A) Mice were subcutaneously injected miR-18a lentivirus- or mock lentivirustransfected MG63 cells. The tumor volumes of the xenografts were monitored weekly $\left({ }^{* *} \mathrm{P}<0.01\right)$. (B and C) The tumors were isolated and weighed at the end of experiment. (B) Three representative tumors from each group are shown. The tumors derived from the miR-18a lentivirus-transfected cells were much smaller than those derived from the mock lentivirus-transfected cells $\left({ }^{* * *} \mathrm{P}<0.01\right)$. (D) There was no difference between the 2 groups as regards body weight during the experiment $(\mathrm{P}>0.05)$.

A

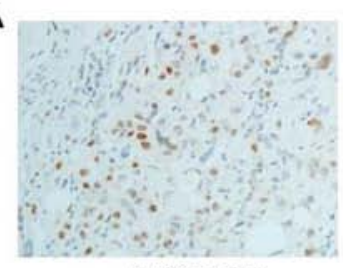

lenti-vector

C

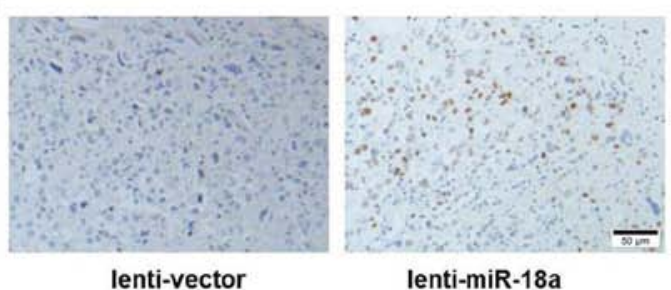

E

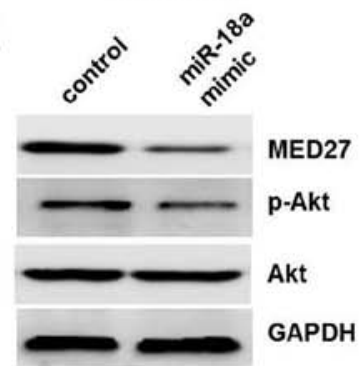

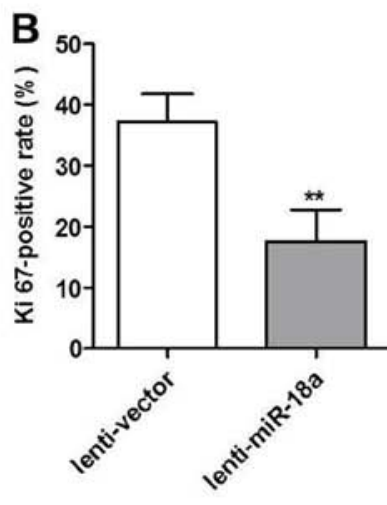
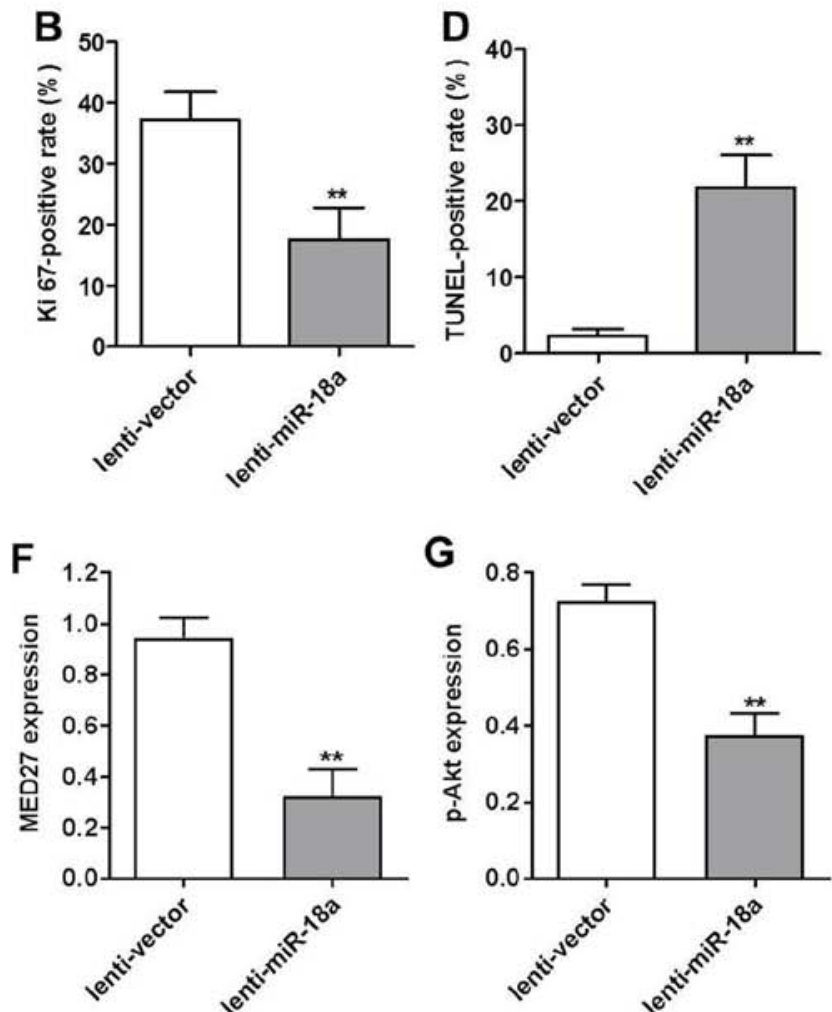

Figure 6. miR-18a increased the number of Ki67 and TUNEL positively stained cells, and inhibits MED27 and p-Akt protein expression in vivo. (A) Immunohistochemical staining of Ki67 expression in the miR-18a lentivirus or mock lentivirus group. (B) The quantification of Ki67 positivity in tumor tissues $\left({ }^{* *} \mathrm{P}<0.01\right)$. (C) TUNEL staining in miR-18a lentivirus or mock lentivirus group. (D) The quantification of TUNEL positivity in tumor tissues $\left({ }^{* *} \mathrm{P}<0.01\right)$. (E) MED27 and p-Akt levels in tumors derived from miR-18a lentivirus- or mock lentivirus-transfected tumors were detected by western blot analysis. ( $F$ and $\mathrm{G})$ Quantification of MED27 and p-Akt expression $\left({ }^{* *} \mathrm{P}<0.01\right.$ compared with the lenti-mock group). 
MED subunits have recently been reported to be involved in tumor growth (41-45), these findings were limited to MED1, 12, 14, 15, 19, 24 and 28, and little was known about the functional role of other subunits of the MED complex in carcinogenesis, including MED27 (46). In this study, we investigated and demonstrated the functional significance of MED27 in osteosarcoma progression. Of note, we found that MED27 protein expression was decreased by transfection with miR-18a mimics along with the downregulation of $\mathrm{p}-\mathrm{AKT}$ expression in OS cells. Taken together, these results may aid to in the elucidation of the role miR-18a, particularly its role in the regulation of OS cell apoptosis and suggest that MED27 may be a novel potential target in the treatment of OS.

\section{Acknowledgements}

Not applicable.

\section{Funding}

No funding was received.

\section{Availability of data and materials}

The datasets used and/or analyzed during the current study are available from the corresponding author on reasonable request.

\section{Authors' contributions}

JD and JL were responsible for sample collection, and the experiment design and execution. LS and PS analyzed and interpreted the experimental data. JD, MH and QC drafted the manuscript, interpreted the experimental data and were involved in the conception of the study. JL reviewed and approved the final draft of this manuscript prior to submission. All authors have read and approved the final manuscript.

\section{Ethics approval and consent to participate}

All the procedures using nude mice were approved by the Xin Hua Hospital Animal Experimental Ethics Committee (Approval no. 201703678).

\section{Consent for publication}

Not applicable.

\section{Competing interests}

The authors declare that they have no competing interests.

\section{References}

1. Ottaviani G and Jaffe N: The epidemiology of osteosarcoma. Cancer Treat Res 152: 3-13, 2009.

2. Whelan J, McTiernan A, Cooper N, Wong YK, Francis M, Vernon $\mathrm{S}$ and Strauss SJ: Incidence and survival of malignant bone sarcomas in England 1979-2007. Int J Cancer 131: E508-E517, 2012.

3. Longhi A, Errani C, De Paolis M, Mercuri M and Bacci G: Primary bone osteosarcoma in the pediatric age: State of the art. Cancer Treat Rev 32: 423-436, 2006.
4. Eilber FR and Rosen G: Adjuvant chemotherapy for osteosarcoma. Semin Oncol 16: 312-322, 1989.

5. Pápai Z, Féja CN, Hanna EN, Sztán M, Oláh E and Szendrôi M: P53 overexpression as an indicator of overall survival and response to treatment in osteosarcomas. Pathol Oncol Res 3: 15-19, 1997.

6. Sakamoto A and Iwamoto Y: Current status and perspectives regarding the treatment of osteo-sarcoma: Chemotherapy. Rev Recent Clin Trials 3: 228-231, 2008.

7. He H, Ni J and Huang J: Molecular mechanisms of chemoresistance in osteosarcoma (Review). Oncol Lett 7: 1352-1362, 2014.

8. Bartel DP: MicroRNAs: Genomics, biogenesis, mechanism, and function. Cell 116: 281-297, 2004.

9. Lim LP, Glasner ME, Yekta S, Burge CB and Bartel DP: Vertebrate microRNA genes. Science 299: 1540, 2003.

10. Lu J, Getz G, Miska EA, Alvarez-Saavedra E, Lamb J, Peck D, Sweet-Cordero A, Ebert BL, Mak RH, Ferrando AA, et al: MicroRNA expression profiles classify human cancers. Nature 435: 834-838, 2005.

11. Tagawa $\mathrm{H}$ and Seto $\mathrm{M}$ : A microRNA cluster as a target of genomic amplification in malignant lymphoma. Leukemia 19: 2013-2016, 2005.

12. He L, Thomson JM, Hemann MT, Hernando-Monge E, Mu D, Goodson S, Powers S, Cordon-Cardo C, Lowe SW, Hannon GJ, et al: A microRNA polycistron as a potential human oncogene. Nature 435: 828-833, 2005.

13. Hayashita Y, Osada H, Tatematsu Y, Yamada H, Yanagisawa K, Tomida S, Yatabe Y, Kawahara K, Sekido Y and Takahashi T: A polycistronic microRNA cluster, miR-17-92, is overexpressed in human lung cancers and enhances cell proliferation. Cancer Res 65: 9628-9632, 2005.

14. Mendell JT: miRiad roles for the miR-17-92 cluster in development and disease. Cell 133: 217-222, 2008.

15. Olive V, Bennett MJ, Walker JC, Ma C, Jiang I, Cordon-Cardo C, Li QJ, Lowe SW, Hannon GJ and He L: miR-19 is a key oncogenic component of mir-17-92. Genes Dev 23: 2839-2849, 2009.

16. Chen YJ, Wu H, Zhu JM, Li XD, Luo SW, Dong L, Liu TT and Shen XZ: MicroRNA-18a modulates P53 expression by targeting IRF2 in gastric cancer patients. J Gastroenterol Hepatol 31: 155-163, 2016.

17. Speidel D: Transcription-independent p53 apoptosis: An alternative route to death. Trends Cell Biol 20: 14-24, 2010.

18. Chen H, Zhou L, Wu X, Li R, Wen J, Sha J and Wen X: The $\mathrm{PI} 3 \mathrm{~K} / \mathrm{AKT}$ pathway in the pathogenesis of prostate cancer. Front Biosci 21: 1084-1091, 2016.

19. Wang KC, Botting KJ, Zhang S, McMillen IC, Brooks DA and Morrison JL: Akt signaling as a mediator of cardiac adaptation to low birth weight. J Endocrinol 233: R81-R94, 2017.

20. Lu Y, Thomson JM, Wong HY, Hammond SM and Hogan BL: Transgenic over-expression of the microRNA miR-17-92 cluster promotes proliferation and inhibits differentiation of lung epithelial progenitor cells. Dev Biol 310: 442-453, 2007.

21. Luo Z, Dai Y, Zhang L, Jiang C, Li Z, Yang J, McCarthy JB, She $X$, Zhang $\mathrm{W}$, Ma J, et al: miR-18a promotes malignant progression by impairing microRNA biogenesis in nasopharyngeal carcinoma. Carcinogenesis 34: 415-425, 2013.

22. Zhang W, Lei C, Fan J and Wang J: miR-18a promotes cell proliferation of esophageal squamous cell carcinoma cells by increasing cylin D1 via regulating PTEN-PI3K-AKT-mTOR signaling axis. Biochem Biophys Res Commun 477: 144-149, 2016.

23. Hsu TI, Hsu CH, Lee KH, Lin JT, Chen CS, Chang KC, Su CY, Hsiao M and Lu PJ: MicroRNA-18a is elevated in prostate cancer and promotes tumorigenesis through suppressing STK4 in vitro and in vivo. Oncogenesis 3: e99, 2014.

24. Wu W, Zhou X, Yu T, Bao Z, Zhi T, Jiang K, Nie E, Wang Y, Zhang $J$ and You Y: The malignancy of miR-18a in human glioblastoma via directly targeting CBX7. Am J Cancer Res 7: 64-76, 2017.

25. Namløs HM, Meza-Zepeda LA, Barøy T, Østensen IH, Kresse SH, Kuijjer ML, Serra M, Bürger H, Cleton-Jansen AM and Myklebost O: Modulation of the osteosarcoma expression phenotype by microRNAs. PLoS One 7: e48086, 2012.

26. Fenger JM, Roberts RD, Iwenofu OH, Bear MD, Zhang X, Couto JI, Modiano JF, Kisseberth WC and London CA: miR-9 is overexpressed in spontaneous canine osteosarcoma and promotes a metastatic phenotype including invasion and migration in osteoblasts and osteosarcoma cell lines. BMC Cancer 16: 784, 2016. 
27. Baumhoer D: Molecular characterization of osteosarcomas. Pathologe 34 (Suppl 2): 260-263, 2013 (In German).

28. Xiao F, Zuo Z, Cai G, Kang S, Gao X and Li T: miRecords: An integrated resource for microRNA-target interactions. Nucleic Acids Res 37 (Database): D105-D110, 2009.

29. Livak KJ and Schmittgen TD: Analysis of relative gene expression data using real-time quantitative PCR and the 2(-Delta Delta C(T)) Method. Methods 25: 402-408, 2001.

30. Ji J and Wang XW: New kids on the block: Diagnostic and prognostic microRNAs in hepatocellular carcinoma. Cancer Biol Ther 8: 1686-1693, 2009.

31. Giráldez MD, Lozano JJ, Ramírez G, Hijona E, Bujanda L, Castells A and Gironella M: Circulating microRNAs as biomarkers of colorectal cancer: Results from a genome-wide profiling and validation study. Clin Gastroenterol Hepatol 11: 681-8.e3, 2013.

32. Lawrie CH: MicroRNA expression in lymphoma. Expert Opin Biol Ther 7: 1363-1374, 2007.

33. Volinia S, Calin GA, Liu CG, Ambs S, Cimmino A, Petrocca F, Visone R, Iorio M, Roldo C, Ferracin M, et al: A microRNA expression signature of human solid tumors defines cancer gene targets. Proc Natl Acad Sci USA 103: 2257-2261, 2006.

34. Schulte JH, Horn S, Otto T, Samans B, Heukamp LC, Eilers UC, Krause M, Astrahantseff K, Klein-Hitpass L, Buettner R, et al: MYCN regulates oncogenic microRNAs in neuroblastoma. Int J Cancer 122: 699-704, 2008.

35. Woods K, Thomson JM and Hammond SM: Direct regulation of an oncogenic micro-RNA cluster by E2F transcription factors. J Biol Chem 282: 2130-2134, 2007.

36. Guil S and Cáceres JF: The multifunctional RNA-binding protein hnRNP A1 is required for processing of miR-18a. Nat Struct Mol Biol 14: 591-596, 2007.

37. Li H, Bian C, Liao L, Li J and Zhao RC: miR-17-5p promotes human breast cancer cell migration and invasion through suppression of HBP1. Breast Cancer Res Treat 126: 565-575, 2011.
38. Nagasawa S, Maeda I, Fukuda T, Wu W, Hayami R, Kojima Y, Tsugawa $\mathrm{K}$ and Ohta T: MED12 exon 2 mutations in phyllodes tumors of the breast. Cancer Med 4: 1117-1121, 2015

39. Shaikhibrahim Z, Menon R, Braun M, Offermann A, Queisser A, Boehm D, Vogel W, Rüenauver K, Ruiz C, Zellweger T, et al: MED15, encoding a subunit of the mediator complex, is overexpressed at high frequency in castration-resistant prostate cancer. Int J Cancer 135: 19-26, 2014

40. Kämpjärvi K, Mäkinen N, Kilpivaara O, Arola J, Heinonen HR, Böhm J, Abdel-Wahab O, Lehtonen HJ, Pelttari LM, Mehine M, et al: Somatic MED12 mutations in uterine leiomyosarcoma and colorectal cancer. Br J Cancer 107: 1761-1765, 2012.

41. Zhao M, Yang X, Fu Y, Wang H, Ning Y, Yan J, Chen YG and Wang G: Mediator MED15 modulates transforming growth factor beta $(\mathrm{TGF} \beta) / \mathrm{Smad}$ signaling and breast cancer cell metastasis. J Mol Cell Biol 5: 57-60, 2013.

42. Jin F, Irshad S, Yu W, Belakavadi M, Chekmareva M, Ittmann MM, Abate-Shen C and Fondell JD: ERK and AKT signaling drive MED1 overexpression in prostate cancer in association with elevated proliferation and tumorigenicity. Mol Cancer Res 11: 736-747, 2013.

43. Li XH, Fang DN and Zeng CM: Knockdown of MED19 by short hairpin RNA-mediated gene silencing inhibits pancreatic cancer cell proliferation. Cancer Biother Radiopharm 26: 495-501, 2011.

44. Chen W, Rogatsky I and Garabedian MJ: MED14 and MED1 differentially regulate target-specific gene activation by the glucocorticoid receptor. Mol Endocrinol 20: 560-572, 2006.

45. Huang CY, Chou YH, Hsieh NT, Chen HH and Lee MF: MED28 regulates MEK1-dependent cellular migration in human breast cancer cells. J Cell Physiol 227: 3820-3827, 2012.

46. Conaway RC and Conaway JW: Function and regulation of the Mediator complex. Curr Opin Genet Dev 21: 225-230, 2011. 